\title{
Clinically Significant Anxiety Symptoms
}

National Cancer Institute

\section{Source}

National Cancer Institute. Clinically Significant Anxiety Symptoms. NCI Thesaurus. Code C150139.

An indication that an individual's anxiety symptoms are or were clinically significant. 